\title{
Sodium taste threshold in children and its relationship to blood pressure
}

J. Arguelles ${ }^{1}$, J.J. Diaz ${ }^{2}$,

I. Malaga ${ }^{2}$, C. Perillan ${ }^{1}$,

M. Costales ${ }^{1}$ and M. Vijande ${ }^{1}$

\author{
${ }^{1}$ Departamento de Biología Funcional, Area de Fisiología, \\ ${ }^{2}$ Departamento de Pediatría, Hospital Universitario Central de Asturias, \\ Universidad de Oviedo, Oviedo, Spain
}

\author{
Correspondence \\ J. Arguelles \\ Departamento de Biologia Funcional \\ Area de Fisiologia \\ Universidad de Oviedo \\ Julian Claveria, 6 \\ 33006 - Oviedo \\ España \\ Fax: +34-985-10-6285 \\ E-mail: jal@uniovi.es
}

Presented at the International Symposium of Neuroendocrinology "Neuroendocrine control of body fluid homeostasis: past, present and future". Ribeirão Preto, SP, Brazil, September 1-3, 2006.

Received October 11, 2006 Accepted March 14, 2007

\begin{abstract}
Popular science has emphasized the risks of high sodium intake and many studies have confirmed that salt intake is closely related to hypertension. The present mini-review summarizes experiments about salt taste sensitivity and its relationship with blood pressure (BP) and other variables of clinical and familial relevance. Children and adolescents from control parents $(\mathrm{N}=72)$ or with at least one essential hypertensive (EHT) parent $(\mathrm{N}=51)$ were investigated. Maternal questionnaires on eating habits and vomiting episodes were collected. Offspring, anthropometric, BP, and salt taste sensitivity values were recorded and blood samples analyzed. Most mothers declared that they added "little salt" when cooking. Salt taste sensitivity was inversely correlated with systolic BP (SBP) in control youngsters ( $\mathrm{r}=$ -0.33; $\mathrm{P}=0.015)$. In the EHT group, SBP values were similar to control and a lower salt taste sensitivity threshold was found. Obese offspring of EHT parents showed higher SBP and C-reactive protein values but no differences in renin-angiotensin-aldosterone system activity. Salt taste sensitivity was correlated with SBP only in the nonobese EHT group $(\mathrm{N}=41 ; \mathrm{r}=0.37 ; \mathrm{P}=0.02)$. Salt taste sensitivity was correlated with SBP in healthy, normotensive children and adolescents whose mothers reported significant vomiting during the first trimester $(\mathrm{N}=18 ; \mathrm{r}=-0.66 ; \mathrm{P}<0.005)$, but not in "non-vomiter offspring" ( $\mathrm{N}=54 ; \mathrm{r}=-0.18$; nonsignificant). There is evidence for a linkage between high blood pressure, salt intake and sensitivity, perinatal environment and obesity, with potential physiopathological implications in humans. This relationship has not been studied comprehensively using homogeneous methods and therefore more research is needed in this field.
\end{abstract}

Key words

- Children

- Hypertension

- Cardiovascular risk factors

- Salt taste sensitivity

- Pregnancy

- Obesity

\section{Introduction}

Blood pressure is a major physiological variable which is maintained within a narrow range by strong homeostatic mechanisms operating throughout the life span. Many factors influence blood pressure and its in- crease has severe deleterious effects.

This article summarizes several studies carried out by our group and others on humans and rats which dealt with some of the factors related to blood pressure homeostasis: salt sensitivity and intake, obesity and perinatal and genetic factors (Figure 1). 
Figure 1. Relations between hypertension and cardiovascular risk factors (CRF). Established (solid lines) and unestablished (dotted lines) relations.

\section{Salt and blood pressure}

Epidemiological research has shown that dietary sodium is an important contributor to the pathogenesis of hypertension (1). While higher blood pressure values are being currently observed in populations with higher salt intake (2), treatment strategies based on lowering salt ingestion have demonstrated their effectiveness in lowering blood pressure (3). Nevertheless, only a limited pro-

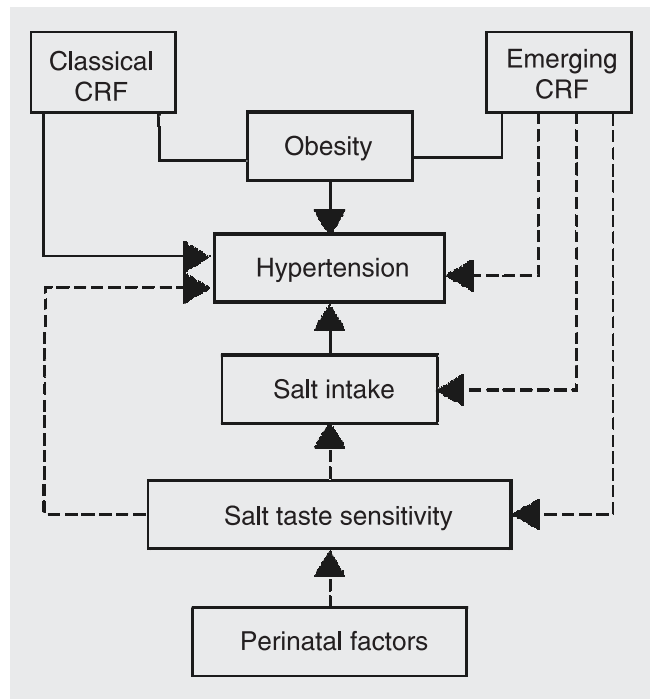

Figure 2. Forced-choice staircase method for the detection of threshold for salt taste sensitivity.

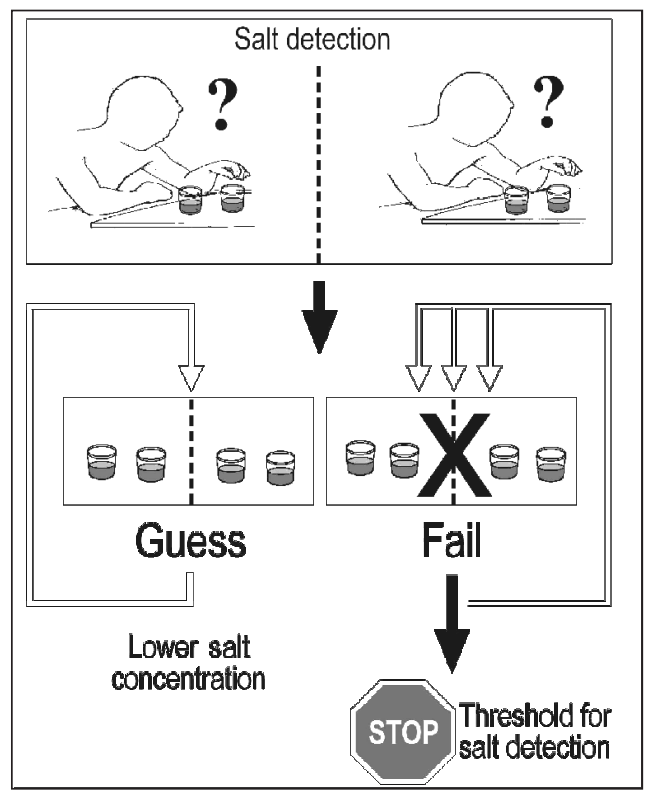

portion of individuals - 5 to $16 \%$ - classified as "salt-dependent" would benefit from dietary salt restriction (4). On the other hand, it seems obvious that the amount of salt intake by an individual should be related in one way or another to his conscious or unconscious ability to recognize it (salt sensitivity). A significant positive relationship between blood pressure and sodium intake has also been reported in pediatric populations (5) and confirmed by meta-analysis (6).

One important aspect to be considered is the possible association between salt perception and blood pressure and its possible variation in normotensive and hypertensive subjects. Because hypertension is typically an adult disease, only minor pathological signs or alterations could be expected during the early years of life. However, these could be significant markers or tracking signals for subjects that eventually will develop hypertension and therefore they would be useful as tools for identifying subjects who could be treated prophylactically or advised about healthy habits early on.

The relationship between salt taste sensitivity (gustatory perception) and blood pressure has been studied to different extents by several investigators, with differing findings. Some of them revealed a positive correlation between salt perception and blood pressure while others did not $(7,8)$.

Our group has studied a cohort of Spanish children and adolescents ( 42 boys and 30 girls) for their familial salt habits, individual salt taste sensitivity and blood pressure (9). Most of the families (84\%) declared that they cook with little salt, which indicates the strong bias of declared salt consumption, perhaps induced by media recommendations on health. These values did not match well the range of fractional sodium excretion found in our subjects which was well within the range of normality $(0.65 \pm 0.03 \%)$.

A behavioral test adapted from Cornsweet (10) was used to assess the salt taste sensitivity, i.e., the lowest $\mathrm{Na}$ concentration de- 
tected when compared to distilled/deionized water (Figure 2). This test consisted of a forced-choice staircase protocol in which subjects received a series of successive pairs of solutions. Within each pair one sample was the unsalted control (distilled deionized water), and the other contained in order one of the following $\mathrm{NaCl}$ solutions: 13.7, 12.2, 10.7, 9.2, 7.7, 6.2, 4.7, 3.2, 1.7, $0.2 \mathrm{mM}$. Each concentration was presented twice. Subjects tasted both samples and chose the saltier within each pair. After a successful detection of the salty cup twice in the same trial, the next less intense stimulus was presented. A failure in any of the trials forced to try the next more intense stimulus (reversal). The procedure was continued until three reversals occurred. The geometric mean of the three reversal concentrations was deemed as the threshold sodium taste sensitivity for the subject.

Sodium taste sensitivity was $4.55 \pm 0.6$ $\mathrm{mM} \mathrm{NaCl}$ (11) and was negatively correlated with systolic blood pressure (SBP, $\mathrm{N}=$ $72 ; \mathrm{r}=-0.33 ; \mathrm{P}<0.01 ;$ Figure 3$)$. Although the mechanism linking these two variables cannot be inferred from these data, the correlation suggests common or related causes for salt taste sensitivity and blood pressure.

\section{Perinatal influences}

Epidemiological and experimental studies have shown that hypertension is mainly a multifactorial disease, with an important genetic component and possibly affected by some perinatal influences. Hypertension is about twice as common in subjects who have one or both hypertensive parents, and it has been estimated that genetic factors account for approximately $30 \%$ of the variation in blood pressure in various human populations (12).

In a sample of 51 normotensive adolescents with at least one essential hypertensive (EHT) parent (13), we found blood pressure values similar to those of the control group, but a lower salt taste sensitivity threshold (Figure 4). When age, sex, and body mass index (BMI) were included as control variables in a multiple regression model, the salt taste sensitivity threshold was significantly correlated with SBP $(B=0.94 ; \mathrm{P}<0.012)$. Interestingly, the regression slope between salt taste sensitivity threshold and SBP in control and in EHT (both statistically sig-
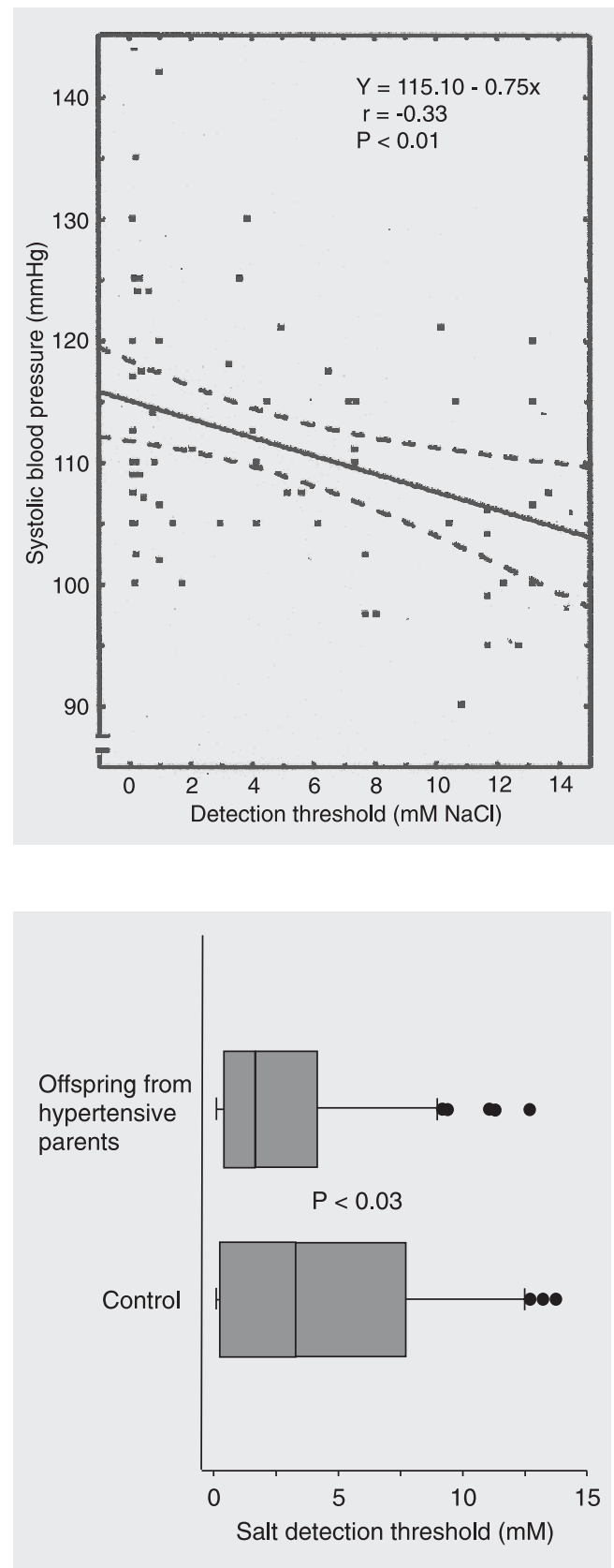

Figure 3. Negative correlation between sodium chloride detection threshold and systolic blood pressure.
Figure 4. Salt detection threshold in the offspring of control and hypertensive parents. Box vertical sides represent the lower and upper quartile (enclosing the middle half of the sample); the sample median, as the vertical line inside; whiskers sprout from the two ends of the box until they reach the sample maximum and minimum, excluding outliers - black symbols (cases with the values between 1.5 and 3 box-lengths from the 75 th or 25 th percentile). 
nificant) had the opposite sign.

Long-lasting effects induced by challenges to body fluid homeostasis during gestation have been tested in different species. Concerning these perinatal influences in pregnant rats, Nicolaidis et al. (14) showed that episodes of extracellular dehydration by polyethylene-glycol administration led to an increased salt appetite in adult offspring. A few years later the same group found an increase in salt appetite in the offspring when acute and repeated mineralofluid losses had occurred during pregnancy after diureticnatriuretic treatment (15). In both cases the subjects underwent dehydration accompanied by an activation of the renin angiotensin system. Leshem (16), working with humans, suggested that episodes of mineralofluid losses during pregnancy or in early infancy could increase salt preference when investigated in late childhood, and that more sensitive tests of salt preference would be required to reveal these influences.

During the last decade, our group has been studying the changes in need-free and stimulated $\mathrm{NaCl}$ intake in newborn and adult rats by manipulations of the levels of $\mathrm{NaCl}$ ingestion during gestation and the early postnatal period (17-20).

Recent studies suggest that maternal angiotensin II may affect the neural substrate in the fetus since angiotensin receptors have been shown to mediate several ontogenic effects including growth, differentiation and apoptosis among others, and especially the AT2 subtype which is abundant in the fetal brain and disappears soon after birth (21).

Human studies focused on salt appetite are complicated by the lack of uniformity in the experimental protocols. Crystal and Bernstein (22) found a greater preference for saltier food in young college students whose mothers reported morning sickness during pregnancy when compared to those with asymptomatic mothers. They also demonstrated that infants from mothers vomiting excessively during early pregnancy drank more concentrated salt solutions than controls and showed less aversive facial reactions to the salty solutions (23).

Results from our work (24) have proved a significant correlation between blood pressure and salt taste perception in a healthy and normotensive adolescent Spanish population $(\mathrm{N}=18 ; \mathrm{r}=-0.66 ; \mathrm{P}<0.005)$ whose mothers reported significant vomiting during the 1st trimester of gestation, i.e., indicating that those subjects with higher SBP values had lower detection thresholds (being more salt sensitive). These data agree with the "Barker hypothesis" (25) applied to the important health field of hypertension, adding new evidence to the emerging programming hypothesis that links early episodes in fetal and neonatal life to the development of disease risk in adult life.

\section{Obesity and cardiovascular risk factors}

The trend towards overweight in adult populations is common in the developed world $(26,27)$. Still more alarming is the progressive rate of obesity detected in pediatric populations in these countries (28-30). All this is related to increasing blood pressure values and even to the prevalence of hypertension and other cardiovascular risk factors, including dyslipidemia, insulin resistance and metabolic syndrome $(31,32)$. The concurrence of some or all of these factors amplifies their potential deleterious cardiovascular effects (33). Although the metabolic syndrome was initially described in adults, it is also often found in children (34).

There are an increasing number of studies indicating that other factors could also be involved in cardiovascular pathology. These are often named as novel or emerging cardiovascular risk factors. A low grade of inflammatory disease affecting the arterial vessels which can be revealed by discrete elevations in C-reactive protein (CRP), only de- 
tectable by using high sensitivity methods, is one of these and could start in childhood (35). CRP is related to age, sex, ethnicity, anthropometric variables, lipid profile, and insulin sensitivity (36), and its values increase in parallel with BMI (37). The discovery of adipocytokines has led to an increased understanding of the role of fatty tissue both in normal homeostasis and in pathological states because they may correspond to an important mechanistic link between obesity and cardiovascular disease (38).

In this respect, we found a $20 \%$ prevalence of obesity in young and adolescent offspring of EHT patients (28 boys and 24 girls, with at least 1 parent with EHT) and studied its relationship to other pathogenic factors for hypertension such as renin-angiotensin-aldosterone system components and CRP (9). Body weight and height significantly correlated with SBP both in obese $(\mathrm{N}=10 ; \mathrm{r}=0.70 ; \mathrm{P}=0.03$ and $\mathrm{r}=0.66 ; \mathrm{P}=$ $0.04)$ and non-obese offspring of EHT patients $(\mathrm{N}=41 ; \mathrm{r}=0.51 ; \mathrm{P}=0.001$ and $\mathrm{r}=$ $0.49 ; \mathrm{P}=0.001)$. In contrast, SBP correlated significantly with BMI only in non-obese subjects $(\mathrm{N}=41 ; \mathrm{r}=0.33 ; \mathrm{P}=0.03)$. Although salt taste threshold was similar in both groups (obese and non-obese), it correlated significantly with SBP only in the nonobese group ( $\mathrm{N}=41 ; \mathrm{r}=0.37 ; \mathrm{P}=0.02)$. Obese offspring of EHT patients showed SBP values $10 \mathrm{mmHg}$ higher than non-obese offspring $(\mathrm{P}=0.004)$ and also higher CRP (when adjusted by age and SBP, $\mathrm{P}=0.024$ ). No differences were found in diastolic blood pressure, kidney function tests, aldosterone, renin activity, or angiotensin-converting enzyme between groups. Therefore, the obese offspring of EHT parents showed a high prevalence of obesity and higher SBP and CRP values than the non-obese offspring but no differences in renin-angiotensin-aldosterone system activity and only limited salt taste sensitivity differences.

On the basis of these considerations, there are some links between high blood pressure, salt intake, perinatal environment and obesity, with potential physiopathological implications in humans, which have not been comprehensively studied using homogeneous methods, thus requiring more research in this field.

\section{References}

1. Keys AE. Coronary heart disease in seven countries. Circulation 1970; 41 (Suppl 4): 1-211.

2. Horan MJ, Blaustein MP, Dunbar JB, Grundy S, Kachadorian W Kaplan NM, et al. NIH report on research challenges in nutrition and hypertension. Hypertension 1985; 7: 818-823.

3. Law MR, Frost CD, Wald NJ. By how much does dietary salt reduction lower blood pressure? III - Analysis of data from trials of salt reduction. BMJ 1991; 302: 819-824.

4. Ducher M, Fauvel JP, Maurin M, Laville M, Maire P, Paultre CZ, et al. Sodium intake and blood pressure in healthy individuals. $J$ Hypertens 2003; 21: 289-294.

5. del Negro MC, Oliver M, Arocha I, Arias F. Arterial hypertension in the adolescent: its possible relation to the salt taste threshold. Rev Esp Cardiol 1992; 45: 227-231.

6. Simons-Morton DG, Obarzanek E. Diet and blood pressure in children and adolescents. Pediatr Nephrol 1997; 11: 244-249.

7. Okoro EO, Uroghide GE, Jolayemi ET. Salt taste sensitivity and blood pressure in adolescent school children in southern Nigeria. East Afr Med J 1998; 75: 199-203.

8. Zumkley H, Vetter H, Mandelkow T, Spieker C. Taste sensitivity for sodium chloride in hypotensive, normotensive and hypertensive subjects. Nephron 1987; 47 (Suppl 1): 132-134.

9. Diaz Martin JJ, Malaga Dieguez I, Arguelles LJ, Dieguez Junquera MA, Vijande VM, Malaga GS. Clustering of cardiovascular risk factors in obese offspring of parents with essential hypertension. Ann Pediatr 2005; 63: 238-243.

10. Cornsweet TN. The staircase-method in psychophysics. Am J Psychol 1962; 75: 485-491.

11. Malaga S, Diaz JJ, Arguelles J, Perillan C, Malaga I, Vijande M. Blood pressure relates to sodium taste sensitivity and discrimination in adolescents. Pediatr Nephrol 2003; 18: 431-434.

12. Beevers G, Lip GY, O'Brien E. ABC of hypertension: The pathophysiology of hypertension. BMJ 2001; 322: 912-916.

13. Arguelles J, Perillan C, Díaz JJ, Málaga I, Vijande M, Malaga S. Alteraciones de la sensibilidad gustativa a la sal en hijos de hipertensos esenciales. Hipertensión 2005; 22: 311-317.

14. Nicolaidis $\mathrm{S}$, Galaverna $\mathrm{O}$, Metzler $\mathrm{CH}$. Extracellular dehydration during pregnancy increases salt appetite of offspring. Am J Physiol 1990; 258: R281-R283.

15. Galaverna O, Nicolaidis S, Yao SZ, Sakai RR, Epstein AN. Endo- 
crine consequences of prenatal sodium depletion prepare rats for high need-free $\mathrm{NaCl}$ intake in adulthood. Am J Physiol 1995; 269: R578-R583.

16. Leshem M. Salt preference in adolescence is predicted by common prenatal and infantile mineralofluid loss. Physiol Behav 1998; 63: 699-704.

17. Arguelles J, Brime JI, Lopez-Sela P, Perillan C, Vijande M. Adult offspring long-term effects of high salt and water intake during pregnancy. Horm Behav 2000; 37: 156-162.

18. Arguelles J, Lopez-Sela P, Brime JI, Costales M, Vijande M. Changes of blood pressure responsiveness in rats exposed in utero and perinatally to a high-salt environment. Regul Pept 1996; 66: 113-115.

19. Perillan C, Costales M, Diaz F, Vijande M, Arguelles J. Thirst changes in offspring of hyperreninemic rat dams. Pharmacol Biochem Behav 2004; 79: 709-713.

20. Vijande M, Brime JI, Lopez-Sela P, Costales M, Arguelles J. Increased salt preference in adult offspring raised by mother rats consuming excessive amounts of salt and water. Regul Pept 1996; 66: $105-108$.

21. Dinh DT, Frauman AG, Johnston Cl, Fabiani ME. Angiotensin receptors: distribution, signalling and function. Clin Sci 2001; 100: 481-492.

22. Crystal SR, Bernstein IL. Morning sickness: impact on offspring salt preference. Appetite 1995; 25: 231-240.

23. Crystal SR, Bernstein IL. Infant salt preference and mother's morning sickness. Appetite 1998; 30: 297-307.

24. Malaga I, Arguelles J, Diaz JJ, Perillan C, Vijande M, Malaga S. Maternal pregnancy vomiting and offspring salt taste sensitivity and blood pressure. Pediatr Nephrol 2005; 20: 956-960.

25. Barker DJ, Osmond C, Golding J, Kuh D, Wadsworth ME. Growth in utero, blood pressure in childhood and adult life, and mortality from cardiovascular disease. BMJ 1989; 298: 564-567.

26. James PT, Rigby N, Leach R. The obesity epidemic, metabolic syndrome and future prevention strategies. Eur J Cardiovasc Prev
Rehabil 2004; 11: 3-8.

27. Anonymous. Executive Summary of the Third Report of the National Cholesterol Education Program (NCEP) expert panel on detection, evaluation, and treatment of high blood cholesterol in adults (adult treatment panel III). JAMA 2001; 285: 2486-2497.

28. Daniels SR, Arnett DK, Eckel RH, Gidding SS, Hayman LL, Kumanyika S, et al. Overweight in children and adolescents: pathophysiology, consequences, prevention, and treatment. Circulation 2005; 111: 1999-2012.

29. Moreno LA, Mesana MI, Fleta J, Ruiz JR, Gonzalez-Gross M, Sarria $A$, et al. Overweight, obesity and body fat composition in Spanish adolescents. The AVENA Study. Ann Nutr Metab 2005; 49: 71-76.

30. Hedley AA, Ogden CL, Johnson CL, Carroll MD, Curtin LR, Flegal KM. Prevalence of overweight and obesity among US children, adolescents, and adults, 1999-2002. JAMA 2004; 291: 2847-2850.

31. Weiss R, Dziura J, Burgert TS, Tamborlane WV, Taksali SE, Yeckel $\mathrm{CW}$, et al. Obesity and the metabolic syndrome in children and adolescents. N Engl J Med 2004; 350: 2362-2374.

32. Eckel RH, Grundy SM, Zimmet PZ. The metabolic syndrome. Lancet 2005; 365: 1415-1428.

33. Lurbe E, Torro I, Alvarez V, Nawrot T, Paya R, Redon J, et al. Prevalence, persistence, and clinical significance of masked hypertension in youth. Hypertension 2005; 45: 493-498.

34. Cruz ML, Goran MI. The metabolic syndrome in children and adolescents. Curr Diab Rep 2004; 4: 53-62.

35. Belay $B$, Belamarich $P$, Racine AD. Pediatric precursors of adult atherosclerosis. Pediatr Rev 2004; 25: 4-16.

36. Koenig W. Update on C-reactive protein as a risk marker in cardiovascular disease. Kidney Int Suppl 2003; S58-S61.

37. Visser M, Bouter LM, McQuillan GM, Wener MH, Harris TB. Lowgrade systemic inflammation in overweight children. Pediatrics 2001; 107: E13.

38. Skilton MR, Celermajer DS. The effects of obesity-related peptides on the vasculature. Curr Vasc Pharmacol 2006; 4: 79-85. 